\title{
EDITORIAL
}

\section{Personalized cancer medicine: era of promise and progress}

$\mathrm{N}$ owhere has the evolution of personalized medicine been more rapid than the field of oncology. Advances in drug development, identification of multiple disease subtypes, and available high-throughput technologies have allowed treatment progress and a better understanding of cancer heterogeneity. Successful personalized medicine requires a global collaborative effort so that diverse populations of cancer patients have the opportunity to participate in clinical trials with the latest innovative drugs. Such a global effort was the basis for the generation of the Worldwide Innovative Networking (WIN) Consortium, initiated by the Institut Gustave Roussy (IGR) and the MD Anderson Cancer Center. The Consortium facilitates the collaboration between clinical cancer centers, industry, patient advocacy groups, government institutions and other stakeholders to enhance patient access to clinical trials and the latest therapies. In July 2010, WIN held its second annual conference, prompting Nature Reviews Clinical Oncology to commission a special focus issue on personalized medicine to highlight the latest advances, opportunities and challenges.

A systems approach to medicine that includes predictive, personalized, preventive and participatory (P4) aspects was first described by Leroy Hood more than 5 years ago to explain the growing trend that medicine is "moving from a reactive to a proactive discipline." The ultimate goal of $\mathrm{P} 4$ medicine is to understand completely the complexity of biological networks that govern the carcinogenic process and to harness this information to provide better patient care. Implementing P4 medicine in clinical practice requires that technical and societal barriers are overcome. In this issue, Leroy Hood and Stephen Friend discuss these challenges and posit that a systems approach to disease will provide new insights via the powerful new diagnostics and therapeutics available. However, the mining and integration of the billions of data points for each individual in order to construct models representing each patient's wellness or disease continuum is key to achieving $\mathrm{P} 4$ medicine. As a testament to the participatory aspect of $\mathrm{P} 4$ medicine, Hood and Friend note that patients are already becoming partners in oncology trials, with trial participation and accrual being driven by patients-the Million Women Study is a pertinent example.

Each cancer is as individual as the patient and continually evolves, and responses to therapies are equally varied - these facts will have a huge impact on the design of clinical trials, especially when thousands of patients are required to detect a small survival or clinical benefit.
The business approach for drug development by pharmaceutical companies, and the FDA approval process, will need a radical change to synergize with the ever increasing pace and complexity of how to test the multitude of drugs in so many diseases. Conducting clinical trials with such high patient numbers to detect relatively small efficacy changes will become prohibitive. Effective collaboration and innovative clinical trial designs are needed.

Researchers at the IGR offer their own perspective and novel suggestions as to how this could be achieved. They suggest patients who could be cured with available treatments should be treated at local, high-volume hospitals. For patients with a high risk of relapse who cannot be cured with conventional agents, newer effective drugs are needed; these patients will be included in trials evaluating new drugs and referred to specialized centers that have the capabilities to use high-throughput technologies for patient management. Tursz et al. envisage biology-driven phase I and II trials that include patient populations that are enriched for the candidate marker. If responses are observed in phase II trials, in situations where a high sensitivity to the drug is demonstrated, they believe it is not ethical to propose further phase III testing but that conditional approval together with data from large phase II trials should be sought to consolidate efficacy and safety findings. This would increase the number of biology-driven phase II trials while decreasing the need for randomized phase III studies.

A key component to implementing this approach will require that biomarker data are accessible, consistently reported and that negative data relating to biomarker outcomes are published. With so many biomarker studies published, selective publication bias-leading to falsepositive findings-and complexities associated with multiple hypothesis testing can distort the true value of a biomarker. Fabrice Andre and coauthors propose that a comprehensive biomarker registry be created for all biomarker studies (including negative data), which would initially focus on using specimens from randomized trials to help reduce bias and allow a standardized, highly accessible and more balanced evaluation of promising candidate biomarkers. Appropriate biomarker testing and independent validation are critical to ensure that the biomarker registry fulfils its full potential, but I welcome the premise behind this initiative and look forward to more progress in the field of personalized medicine.

doi:10.1038/nrclinonc.2011.14

\section{Effective collaboration \\ and \\ innovative clinical trial designs are needed 77}

Lisa Hutchinson is the Chief Editor of Nature Reviews Clinical Oncology.

Competing interests The author declares no competing interests. 